\title{
Developing Machine-Learning Prediction Algorithm for Bacteremia in Admitted Patients
}

This article was published in the following Dove Press journal:

Infection and Drug Resistance

\author{
Ebrahim Mahmoud (D) \\ Mohammed Al Dhoayan ${ }^{2,3}$ \\ Mohammad Bosaeed ${ }^{1,4,5}$ \\ Sameera Al Johani (iD) 5,6 \\ Yaseen $\mathrm{M} \mathrm{Arabi}^{5,7}$ \\ 'Department of Infectious Disease, \\ Department of Medicine, King Abdulaziz \\ Medical City, Riyadh, Saudi Arabia; \\ ${ }^{2}$ Department of Health Informatics, \\ $\mathrm{CPHHI}$, King Saud Bin Abdulaziz \\ University for Health Sciences, Riyadh, \\ Saudi Arabia; ${ }^{3}$ Data and Business \\ Intelligence Management Department, \\ ISID, King Abdulaziz Medical City, Riyadh, \\ Saudi Arabia; ${ }^{4}$ King Abdullah \\ International Medical Research Center \\ (KAIMRC), Riyadh, Saudi Arabia; \\ ${ }^{5}$ College of Medicine, King Saud Bin \\ Abdulaziz University For Health Sciences, \\ Riyadh, Saudi Arabia; ${ }^{6}$ Department of \\ Pathology \& Laboratory Medicine, King \\ Abdulaziz Medical City, Riyadh, Saudi \\ Arabia; ${ }^{7}$ Department of Intensive Care, \\ King Abdulaziz Medical City, Riyadh, \\ Saudi Arabia
}

Purpose: Bloodstream infection among hospitalized patients is associated with serious adverse outcomes. Blood culture is routinely ordered in patients with suspected infections, although $90 \%$ of blood cultures do not show any growth of organisms. The evidence regarding the prediction of bacteremia is scarce.

Patients And Methods: A retrospective review of blood cultures requested for a cohort of admitted patients between 2017 and 2019 was undertaken. Several machine-learning models were used to identify the best prediction model. Additionally, univariate and multivariable logistic regression was used to determine the predictive factors for bacteremia.

Results: A total of 36,405 blood cultures of 7157 patients were done. There were 2413 $(6.62 \%)$ positive blood cultures. The best prediction was by using NN with the high specificity of $88 \%$ but low sensitivity. There was a statistical difference in the following factors: longer admission days before the blood culture, presence of a central line, and higher lactic acid-more than $2 \mathrm{mmol} / \mathrm{L}$.

Conclusion: Despite the low positive rate of blood culture, machine learning could predict positive blood culture with high specificity but minimum sensitivity. Yet, the SIRS score, qSOFA score, and other known factors were not good prognostic factors. Further improvement and training would possibly enhance machine-learning performance.

Keywords: bacteremia, blood culture prediction, machine learning, predictive medicine

\section{Introduction}

Bloodstream infection (BSI) is associated with adverse outcomes, including serious complications and increased mortality. ${ }^{1,2}$ Early detection of BSI is important because its absence may result in inappropriate initial therapy and lead to an increase in overall mortality. ${ }^{3}$

Though blood culture is routinely ordered for patients with suspected infections, only a small proportion of cultures yield true-positive results. Studies have demonstrated that as many as $90 \%$ of all blood cultures do not show growth of any organism. ${ }^{4}$ Published guidelines do not clearly state when blood cultures should be drawn. Usually, clinician depends on their assessment to order for blood culture if the patient has a fever or a suspected endocarditis, or defined infectious syndromes like central line-associated bloodstream infection (CLABSI).

The field of machine learning is advancing rapidly and, through occurrences and

Correspondence: Ebrahim Mahmoud

Division of Infectious Diseases,

Department of Medicine, King Abdulaziz

Medical City, Riyadh, Riyadh, Saudi Arabia

Tel +96650008I418

Emailemahmoud85@gmail.com experience continuously learns and improves its skill and decision-making ability. We hypothesize that machine learning would improve the accuracy of predicting bacteremia. This study was aimed at using machine-learning algorithms with data 
or factors that are routinely collected while admitting patients to the hospital to calculate the probability that a requested blood culture will return a positive result.

\section{Patients and Methods}

\section{Source of Data}

The study was conducted at King Abdulaziz Medical City (KAMC), which is a tertiary care center in Riyadh, Saudi Arabia with a capacity of over 1500 beds. The hospital uses the health information system "BEST Care". It is an electronic health record (EHR) that contains all the information about a patient, such as data on medication and physicians' orders.

\section{Participants}

In this retrospective cohort study of blood cultures of admitted, adult patients (age more than 14 years) between July 2017 to July 2019. A flow chart is provided in Figure S1; Supplement 1. The exclusion criteria were repeated tests for the same patient on the same day and patients with "hematological malignancy and organ transplant recipients". Also, patients who were admitted to the ICU after $48 \mathrm{~h}$ from their initial admission to the hospital were excluded. Nevertheless, patients who were shifted to the ICU within the first $48 \mathrm{~h}$ of their admission to the hospital were included. Data related to the patients' blood culture results and other independent variables were acquired and analyzed to derive and validate an algorithm that could predict positive blood culture.

\section{Study Outcome}

The outcome was based on positive or negative blood cultures. Contaminant organisms according to Clinical Laboratory \& Standards Institute (CLSI) Guidelines were considered as negative. Blood culture, whether positive or negative, was tracked from the time of collection to look for the vital signs and other laboratory investigations before the time of the collection by 12-24 hours.

Several descriptive and predictive analytical techniques were used to detect data patterns and establish associations between positive blood cultures and the routinely collected data.

\section{Predictors}

The list of covariates was included in the data as follows:

The following factors were considered to be predictor variables: age, admittance diagnosis, length of stay before blood sample collection, co-morbidities, presence of central line-Foley catheter and tracheostomy at the time of blood culture request, and receiving antibiotics $24 \mathrm{~h}$ before the blood sample was taken. All of these variables were collected before blood culture collection. Vital signs: Temperature, heart rate, systolic and diastolic blood pressure, respiratory rate, and Glasgow Coma scale. Laboratory testing: WBC count, platelet count, creatinine level, lactic acid level, C-reactive protein (CRP), and procalcitonin. SIRS and qSOFA scores were also included in the analysis.

\section{Missing Variables}

After all the data pre-processing was conducted, missing values were review. To remedy for missing values, two techniques were used. First, to drop out any record with any missing data point. Second, since most of the missing values were missing completely at random (MCAR), k-Nearest Neighbors imputation was used to impute these missing values with the 3 nearest neighbors and a uniform weight function. ${ }^{5}$

All used ML algorithms were performed on data with and without missing data imputation and their performance was compared to measure the effect of missing data on the performed analyses. Furthermore, the interquartile range (IQR) rule was used to detect and remove any outliers. ${ }^{6}$

The last step in the pre-processing was to balance the distribution between negative and positive blood cultures in the outcome variable to avoid any bias in the ML models' predictions. To do so, the training data were subjected to Synthetic Minority Over-Sampling Technique (SMOTE) using the Imbalanced-learn package in Python 3.7.,8 Missing variables numbers are provided in Table S2; Supplement 3.

\section{Definitions}

\section{Contamination}

The following bacterial pathogens were recognized as contaminants according to Clinical Laboratory \& Standards Institute (CLSI) Guidelines, namely, coagulasenegative staphylococci, Corynebacterium spp. ("diphtheroid"), Propionibacterium spp, Aerococcus spp, Micrococcus spp, or Bacillus spp. Cultures showing their presence were considered as negative blood culture result. It is to be noted that blood cultures with two organisms or more were considered as positive if an organism that is not considered as a contaminant was present.

\section{Statistics and Machine Learning}

To achieve the objective of this study, predictive analytics were used to develop classification models that could 
differentiate between positive and negative blood cultures using the data elements described above. Since the outcome of the blood cultures was either positive or negative, multiple binary ML algorithms were trained and validated to classify each blood culture to either positive or negative. The followed approach was to train multiple models on the same dataset and compare their performances. The first model was built using Random Forest (RF) with 100 estimators and maximum depth of 2 levels. ${ }^{9}$ The second model was built using Logistic Regression (LR, aka logit, MaxEnt) classifier, which implements regularized logistic regression using the "liblinear" solver. ${ }^{10}$

The third was built using Decision Trees (DT) with a maximum depth of 2 levels. ${ }^{11}$ The fourth model was built using Naive Bayes classifier for multivariate Bernoulli models (NB). ${ }^{12}$ The fifth was using Neural Networks (NN) with 7 hidden layers with a dropout of $20 \%$ to control for over fitting and a learning rate of $0.1{ }^{13}$ All layers had Elu activation function except for the last layer which had sigmoid activation function for binary outcome. The sixth model was C-Support Vector Machine Classification (SVM) with a Radial Basis Function (RBF) kernel, and 0.031 gamma. ${ }^{14}$ All these models were subjected to multiple hyperparameter tunings to select the best setup for each algorithm. All of these models (Except for NN) were developed using Scikit-learn package in Python 3.7. ${ }^{15}$

Furthermore, univariate logistic regression models were used to detect patterns and associations between the independent variables and the outcome of the blood cultures. All variables that were found to be associated with blood culture outcome at a level of significance p-value 0.05 were included in a multiple logistic regression model with a level of significance at p-value 0.05 as well.

\section{Results}

\section{Participants}

Between July 2017 and July 2019, 36,405 blood cultures were requested for 7157 admitted patients. The final analysis for the prediction model included ( $\mathrm{n}=21,073$ cultures).

Their mean age was 61.5 years, with almost equal numbers of males and females. The mean hospital length of stay was 18.9 days before blood culture was requested. Almost one-fifth of the patients had a central line at the time of blood culture request $(18.35 \%)$ and urinary tract infections were labeled in $20.86 \%$ of the patients. Concerning comorbidities, $11.45 \%$ had heart failure and $13.63 \%$ of the patients underwent surgical procedures within 14 days of blood culture requests. The other demographics are listed in Table 1.
Table I Characteristics of Blood Cultures Episodes Included in Analysis $(n=21,073)$

\begin{tabular}{|c|c|c|}
\hline & $\begin{array}{l}\text { Mean } \\
(n=21,073)\end{array}$ & SD \\
\hline Age, y & 61.51 & 21.15 \\
\hline Gender; male (\%) & 52.20 & 0.49 \\
\hline Length of hospitalization "Before test", in days & 18.90 & 36.27 \\
\hline Antibiotics use* $(\%)$ & 88.96 & 0.31 \\
\hline Surgery** (\%) & 13.63 & 0.34 \\
\hline Liver cirrhosis (\%) & 4.90 & \\
\hline End stage renal disease (\%) & 5.61 & \\
\hline Heart failure (\%) & 11.45 & \\
\hline Stroke $(\%)$ & 7.65 & \\
\hline Urinary tract infection (\%) & 20.86 & \\
\hline \multicolumn{3}{|l|}{ Clinical variables } \\
\hline Respiratory rate, breath/min & 21.65 & 3.86 \\
\hline $\mathrm{SBR}$ in $\mathrm{mm} \mathrm{Hg}$ & 121.35 & 19.99 \\
\hline Temperature, ${ }^{\circ} \mathrm{C}$ & 37.08 & 0.62 \\
\hline $\mathrm{DBR}$ in $\mathrm{mm} \mathrm{Hg}$ & 64.34 & 13.08 \\
\hline Heart rate, beats $/ \mathrm{min}$ & 92.12 & 17.82 \\
\hline GCS & 12.24 & 3.17 \\
\hline Temperature $\geq 39^{\circ} \mathrm{C}(\%)$ & 1.18 & 0.10 \\
\hline Temperature $\geq 38{ }^{\circ} \mathrm{C}(\%)$ & 8.77 & 0.28 \\
\hline Central lines catheter (\%) & 18.35 & 0.38 \\
\hline Foley catheter (\%) & 19.49 & 0.39 \\
\hline Tracheostomy (\%) & 0.15 & 0.03 \\
\hline \multicolumn{3}{|l|}{ Lab/score variables } \\
\hline Platelet count, (109/L) & 247.62 & 154.46 \\
\hline White blood cell count, (109/L) & 9.998 & 5.85 \\
\hline Albumin level, g/liter & 30.06 & 5.50 \\
\hline Creatinine level, umol/L & 131.88 & 114.24 \\
\hline Sodium level, serum, $\mathrm{mEq} / \mathrm{L}$ & 135.79 & 5.66 \\
\hline Lactic acid equal or more $2(\mathrm{mmol} / \mathrm{L})(\%)$ & 12.98 & 0.33 \\
\hline CRP level $=$ or $>50 \mathrm{mg} / \mathrm{L}(\%)$ & 12.00 & 0.32 \\
\hline Procalcitonin level $=$ or $>1(\%)$ & 1.72 & 0.13 \\
\hline SIRS & 1.55 & 1.04 \\
\hline qSOFA score & 1.03 & 0.86 \\
\hline \multicolumn{3}{|l|}{ Clinical outcome } \\
\hline 30 days mortality after blood culture request & $11.73 \%$ & \\
\hline
\end{tabular}

Notes: $* 24$ hours before blood culture was obtained. **Within 14 days before blood culture was obtained. Systemic Inflammatory Response Syndrome (SIRS) is the occurrence of at least two of the following criteria: fever $>38.0^{\circ} \mathrm{C}$ or hypothermia $<36.0^{\circ} \mathrm{C}$, tachycardia $>90$ beats/minute, tachypnea $>20$ breaths/minute, leukocytosis $>12 * 109 / /$ or leucopenia $<4 * 109 / 1 .{ }^{26}$ Respiratory rate_22/min, GCS $<15$ and Systolic blood pressure_100mmHg. ${ }^{35}$

Abbreviations: SBR, systolic blood pressure; DBR, diastolic blood pressure; GCS, Glasgow Coma Scale; qSOFA, quick SOFA score.

The majority of the patients were on antibiotics the day before collection (89.96\%). Their mean (Quick SOFA) qSOFA 
Table 2 Microbiology of True Bacteremia

\begin{tabular}{|l|l|l|}
\hline Organism & Number & Percentage \\
\hline Gram Positive & 246 & $10.19 \%$ \\
\hline Staphylococcus aureus & 199 & $8.25 \%$ \\
Enterococcus species & 74 & $3.07 \%$ \\
Streptococcus viridans & 32 & $1.33 \%$ \\
Other & \multicolumn{2}{|l}{} \\
\hline Gram Negative & 283 & $11.73 \%$ \\
\hline Escherichia coli & 230 & $9.53 \%$ \\
Pseudomonas species & 135 & $5.59 \%$ \\
Enterobacter species & 107 & $4.43 \%$ \\
Acinetobacter species & 533 & $22.09 \%$ \\
Klebsiella species & 50 & $2.07 \%$ \\
Serratia species & 174 & $7.21 \%$ \\
Other & 350 & $14.50 \%$ \\
Candida species &
\end{tabular}

score was 1 , and their mean SIRS score was 1.55. Among $12 \%$ of the patients, lactic acid was more than $2 \mathrm{mmol} / \mathrm{L}$.

Their vital signs were near-normal except for the higher mean of the respiratory rate of 21 breaths $/ \mathrm{min}$. Only $8.77 \%$ of the patients had a temperature of $38^{\circ} \mathrm{C}$ or more, at the time of blood culture request. The presence of higher temperatures, more than $39^{\circ} \mathrm{C}$ was the trigger for blood culture was in $1.18 \%$ of the patients.

\section{Positive Blood Culture and Microbiology}

The number of total true positive blood cultures was 2413 and those with contaminations were 1829. Table 2 lists the organisms found in positive blood cultures. The most common pathogen was Enterobacteriaceae (Klebsiella, Pseudomonas, and E. coli). These collectively accounted for $1525(43 \%)$ of total cultures. Candidemia was found in $350(14.5 \%)$ cultures and was more frequent than Staph. aureus found in $246(10.19 \%)$ cultures. The distribution of positive blood cultures suggests that one-third of the positive blood cultures resulted from communityacquired infections (occurred in the first $72 \mathrm{~h}$ of the admission), while (43\%) occurred after 16 days of admission.

\section{Performance of the Models and Predictor}

Table 3 lists the performance of each model. The highest specificity achieved by NN was $89 \%$, with a sensitivity of $17 \%$. The best sensitivity was by Logistic regression $(31 \%)$ with a specificity of $(73 \%)$. Performance of nonimputed machine learning models is provided in Table S1; Supplement 2. Although SVM scored 100\% specificity, it scored zero on sensitivity, which rendered the high specificity null. Since the SVM and NN are considered blackbox algorithms, it is impossible to identify the relative importance of the independent variables to the prediction of positive blood culture. For this reason, in addition to machine learning, we undertook the examination of univariate relationships then multivariate analysis to identify the eligible covariates (see Table 4).

The following factors are believed to be most significantly associated:

Length of hospitalization more than 16 days (OR,1.88; 95\% CI, 1.70-2.08), presence of central line catheter (OR,1.87; 95\% CI, 1.67-2.09), lactic acid more than 2 (OR, 1.53; 95\% CI, 1.34-1.75) and Glasgow Coma Scale score (OR,1.23; 95\% CI, 1.11-1.36).

Among the vital signs, only temperature was considered to be statistically significant (OR,1.23; 95\% CI, 1.14-1.33): temperature at $38^{\circ} \mathrm{C}$ or more $(\mathrm{OR}, 1.50 ; 95 \% \mathrm{CI}$, $1.28-1.75)$ and temperature at $39^{\circ} \mathrm{C}$ or more (OR,1.62; 95\% CI, 1.11-2.38). Meanwhile, several factors such as being on antibiotics or leukocyte count did not show statistical significance. Nevertheless, the following factors were found to affect machine-learning performance:

Demographics and comorbidities: Age, antibiotics use, surgery within 14 days, Central Lines catheter, and length of hospitalization before blood culture test. Vital signs:

Table 3 Machine Learning Models Performance

\begin{tabular}{|c|c|c|c|c|c|}
\hline Algorithm & Accuracy & Precision & Specificity & Sensitivity & AUC \\
\hline Decision tree & 0.80 & 0.09 & 0.86 & 0.15 & 0.51 \\
\hline SVM & 0.91 & I & I & 0 & 0.50 \\
\hline Random forest & 0.75 & 0.11 & 0.79 & 0.30 & 0.54 \\
\hline Logistic regression & 0.70 & 0.09 & 0.73 & 0.31 & 0.52 \\
\hline NN & 0.82 & 0.12 & 0.88 & 0.17 & 0.53 \\
\hline NB & 0.75 & 0.11 & 0.80 & 0.28 & 0.54 \\
\hline
\end{tabular}

Abbreviations: SVM, support vector machine; NN, neural networks; NB, naïve Bayes. 
Table 4 Independent Univariate Predictors of Positive Blood Culture Results

\begin{tabular}{|c|c|c|c|c|c|c|}
\hline & \multicolumn{2}{|c|}{ Positive $(n=1696)$} & \multicolumn{2}{|c|}{ Negative $(n=19,377)$} & \multicolumn{2}{|c|}{ Univariate Analysis } \\
\hline & Mean & SD & Mean & SD & OR $(95 \% \mathrm{Cl})$ & $P$ value \\
\hline Age, y & 60.33 & 20.75 & 61.61 & 21.18 & $0.99(0.99-0.99)$ & 0.01 \\
\hline Length of hospitalization "Before test", in days & 29.89 & 48.68 & 17.94 & 34.81 & $1.00(1.00-1.00))$ & $<0.001$ \\
\hline Antibiotics use (\%) & 87.91 & 0.32 & 89.05 & 0.31 & $0.89(0.76-1.04)$ & 0.14 \\
\hline GCS & 11.17 & 4.09 & 12.01 & 3.73 & $1.23(1.11-1.36)$ & $<0.001$ \\
\hline Surgery & 13.20 & 0.33 & 13.67 & 0.34 & $0.96(0.82-1.1 \mathrm{I})$ & 0.58 \\
\hline Platelet Count, (109/L) & 214.33 & 151.65 & 250.54 & 154.36 & $0.99(0.99-0.99)$ & $<0.001$ \\
\hline White Blood Cell Count, (109/L) & 9.97 & 6.34 & 10.00 & 5.81 & $0.99(0.99-1.00)$ & 0.83 \\
\hline Albumin Level, g/liter & 29.52 & 5.64 & 30.11 & 5.49 & $0.98(0.97-0.98)$ & $<0.001$ \\
\hline Creatinine Level, umol/L & 141.23 & 122.66 & 131.06 & 113.44 & $1.00(1.00-1.00)$ & $<0.001$ \\
\hline Sodium Level, Serum, mEq/L & 135.66 & 5.83 & 135.80 & 5.64 & $0.99(0.98-1.00)$ & 0.31 \\
\hline Respiratory rate, breath/min & 21.97 & 4.18 & 21.62 & 3.83 & $1.02(1.00-1.03)$ & $<0.001$ \\
\hline SBR in $\mathrm{mm} \mathrm{Hg}$ & 118.61 & 20.69 & 121.59 & 19.91 & $0.99(0.98-0.99)$ & $<0.001$ \\
\hline Temperature, ${ }^{\circ} \mathrm{C}$ & 37.16 & 0.70 & 37.07 & 0.61 & $1.23(1.14-1.33)$ & $<0.001$ \\
\hline $\mathrm{DBR}$ in $\mathrm{mm} \mathrm{Hg}$ & 63.10 & 14.02 & 64.45 & 12.99 & $0.99(0.98-0.99)$ & $<0.001$ \\
\hline Heart rate, beats/min & 95.49 & 19.16 & 91.83073 & $|7.6707|$ & $1.01(1.00-1.01)$ & $<0.001$ \\
\hline Central Lines catheter (\%) & 28.41 & 0.45 & 17.46 & 0.37 & $1.87(1.67-2.09)$ & $<0.001$ \\
\hline Foley Catheter (\%) & 17.92 & 0.38 & 19.63 & 0.39 & $0.89(0.78-1.01)$ & 0.08 \\
\hline Gender, Female (\%) & 43.63 & 0.49 & 48.15 & 0.49 & $0.83(0.75-0.92)$ & $<0.001$ \\
\hline Lactic Acid= or $>2$ (mmol/L) $(\%)$ & 18.04 & 0.38 & 0.12 & 33.11 & $1.53(1.34-1.75)$ & $<0.001$ \\
\hline CRP Level $=$ or $>50 \mathrm{mg} / \mathrm{L}(\%)$ & 13.03 & 0.33 & 0.11 & 32.39 & $1.10(0.95-1.28)$ & 0.17 \\
\hline Procalcitonin Level = or $>$ I (\%) & 2.18 & 0.14 & 1.68 & 0.12 & $1.30(0.92-1.83)$ & 0.13 \\
\hline SIRS & 1.72 & 1.06 & 1.54 & 1.04 & $1.18(1.12-1.24)$ & $<0.001$ \\
\hline qSOFA & 1.17 & 0.90 & 1.02 & 0.85 & $1.22(1.15-1.29)$ & $<0.001$ \\
\hline Temperature $\geq 39^{\circ} \mathrm{C}(\%)$ & 1.82 & 0.13 & 1.13 & 0.10 & $1.62(1.11-2.38)$ & 0.01 \\
\hline Temperature $\geq 38{ }^{\circ} \mathrm{C}(\%)$ & 12.20 & 0.32 & 8.47 & 0.27 & $1.50(1.28-1.75)$ & $<0.001$ \\
\hline Positive SIRS score (\%) & 57.72 & 0.49 & 50.37 & 0.49 & $1.34(1.21-1.48)$ & $<0.001$ \\
\hline Positive qSOFA (\%) & 37.44 & 0.48 & 29.87 & 0.45 & $1.40(1.26-1.55)$ & $<0.001$ \\
\hline Length of hospitalization "Before test", 16 days (\%) & 43.75 & 0.49 & 29.22 & 0.45 & $1.88(1.70-2.08)$ & $<0.001$ \\
\hline
\end{tabular}

Notes: Systemic inflammatory response syndrome (SIRS) is the occurrence of at least two of the following criteria: fever $>38.0^{\circ} \mathrm{C}$ or hypothermia $<36.0^{\circ} \mathrm{C}$, tachycardia $>90$ beats/minute, tachypnea $>20$ breaths/minute, leukocytosis $>12 * 109 / /$ or leucopenia $<4 * 109 / 1{ }^{26}$ Respiratory rate_22/min, GCS <15 and Systolic blood pressure_100mmHg. ${ }^{35}$ Abbreviations: SBR, systolic blood pressure; DBR, diastolic blood pressure; GCS, Glasgow Coma Scale; qSOFA, quick SOFA score.

Respiratory rate, systolic blood pressure, temperature, diastolic blood pressure, heart rate, and temperature. Laboratory test: White blood cell count, sodium level, platelet count, albumin level, and creatinine level.
The Scores of SIRS (OR,1.18; 95\% CI, 1.12-1.24), and qSOFA (OR,1.22; 95\% CI, 1.15-1.29), or their performance whether positive or negative (score 2 or more) (OR,1.34; 95\% CI, 1.21-1.48) (OR,1.40; 95\% CI, 
Table 5 Independent Multivariate Predictors of Positive Blood Culture Results

\begin{tabular}{|l|l|l|l|}
\hline & OR $(\mathbf{9 5} \% \mathbf{C I})$ & P value & Z Score \\
\hline Central line catheter & $1.37(1.21-1.55)$ & 0 & 5.01 \\
Lactic acid= or $>2$ (mmol/L) & $1.31(1.13-1.52)$ & 0.0002 & 3.68 \\
Length of hospitalization "Before test", I6 days & $1.30(1.14-1.48)$ & 0.0001 & 3.97 \\
\hline
\end{tabular}

1.26-1.55) respectively, were potential univariate predictors of bacteremia. However, they were not considered statistically significant in multivariate analysis. In the final stepwise logistic regression (Table 5), the significant predictors of bacteremia were the length of hospitalization (OR,1.30; 95\% CI, 1.14-1.48), central line (OR, 1.37; 95\% CI, 1.21-1.55) and lactic acid more than 2 (OR, 1.31; 95\% CI, 1.13-1.52).

\section{Discussion}

In this largest analysis of the predictors of the positive blood culture using machine learning, there were nearly 34,000 negative blood cultures withdrawn during the study period, highlighting the financial waste and the unnecessary burden on the microbiology lab. However, those findings are little less than the literature rate of positivity between $(8-10 \%)^{16-18}$ for such a serious infection that has a crude 30 -days mortality rate between $13 \%-21 \%$. ${ }^{1}$ This attempt at predicting positive blood culture is not a novel idea. ${ }^{16,18-21}$ However, the previous work focused mainly on communityacquired bacteremia setting (patients reporting to the emergency room), and the result was limited by low specificity. ${ }^{18}$

In our model, very high specificity was achieved but the sensitivity was low. Thus, our model would show higher false-negative results and missing true cases of bacteremia being labeled as negative, although the ability to rule out the disease was high. We believe the limited sensitivity and performance are likely to be related to the following:

1. The drawing of blood culture involves several steps and factors including the volume of the sample, which could affect the result. The volume of the blood sample has been noted in the old literature as the single most important factor for positivity and that holds in the era of highly automated blood culture machines based on the higher positive rate in the patients with higher APACHE II scores. ${ }^{22}$ Such factors are relevant in clinical practice and of value, and may explain the discordant result (positive and negative blood culture results, both collected at the same time: $(n=743$ episodes $)$, in our analysis.

2. The Heterogeneity implicated

A) The variable risk for bacteremia, which has been postulated by different studies depending on the different infectious syndromes (low risk in isolated fever, but as high as $50 \%$ in discitis, meningitis, and catheter-associated blood-stream infection). ${ }^{23}$ Thus, to cohort all of those patients in the same category assuming all patients have the same risk of bacteremia is likely to affect the machinelearning ability to predict because the risk needs to be classified based on various syndromes.

B) Variability among the patient population and the pathogens causing bacteremia. Therefore, bacteremia in the first 48 hours of admission is different from hospitalacquired infections whether in terms of the pathogen involved, risk factors, and site of infection. ${ }^{24}$ Furthermore, different etiologies of bacteremia and pathogens (grampositive- gram-negative and candidemia) shown to make a difference to the machine-learning model's performance. The distribution of blood culture through the admission period is provided in Figure S2; Supplement 4.

C) The variable host response to bacteremia ranges between the extremes of stable hemodynamic or shock resulting in multiorgan failure. This was strongly demonstrated by the SIRS and qSOFA score among positive blood cultures; $42 \%$ had a negative SIRS score $<2$ and $63 \%$ had negative qSOFA (Figure 1) in contrast with the previous works that showed good association with the SIRS score. ${ }^{19,25}$ Interestingly, since the introduction of the term SIRS in $1992,{ }^{26}$ it was clear that bacteremia could intersect with sepsis and/or SIRS. This observation still holds. Thus, sensitivity in predicting sepsis is low and this was one of the reasons for redefining sepsis as occurring in 1 out of 8 patients (12.5\%), and multiorgan failure (MOF) did not meet at least two if the SIRS criteria. ${ }^{27,28}$ Furthermore, the machine-learning model to predict sepsis by Giannini et al had a similar problem of low sensitivity. $^{29}$ 


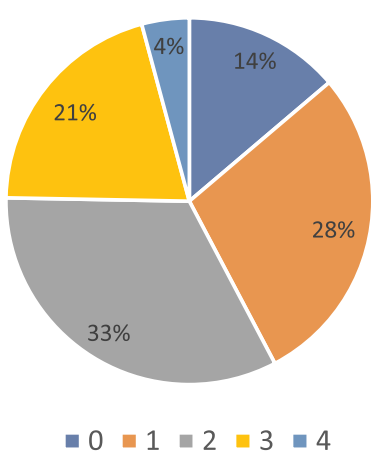

A SIRS Score among Positive blood cultures

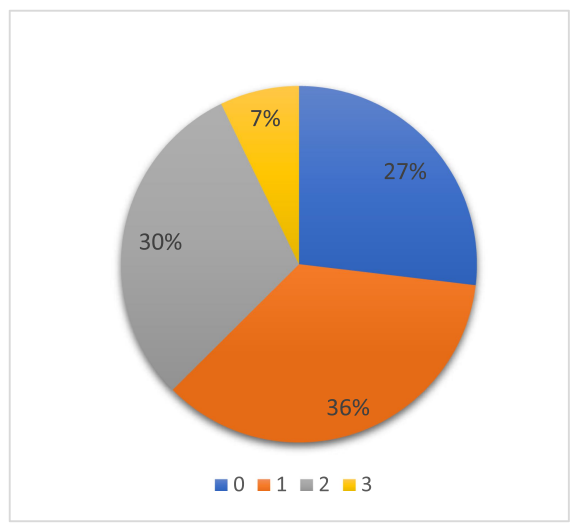

C qSOFA among positive blood cultures

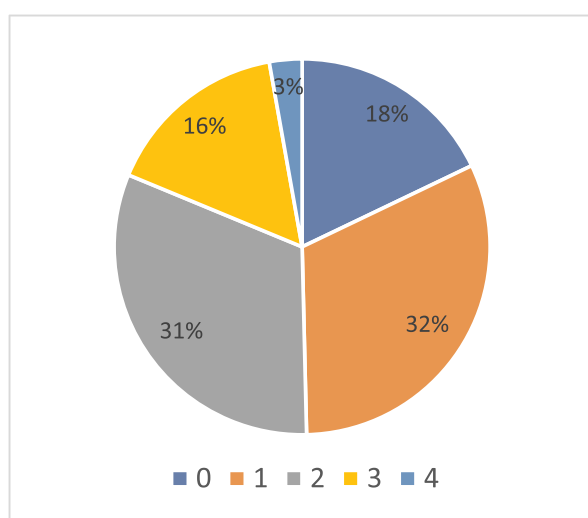

B SIRS Score among negative blood cultures

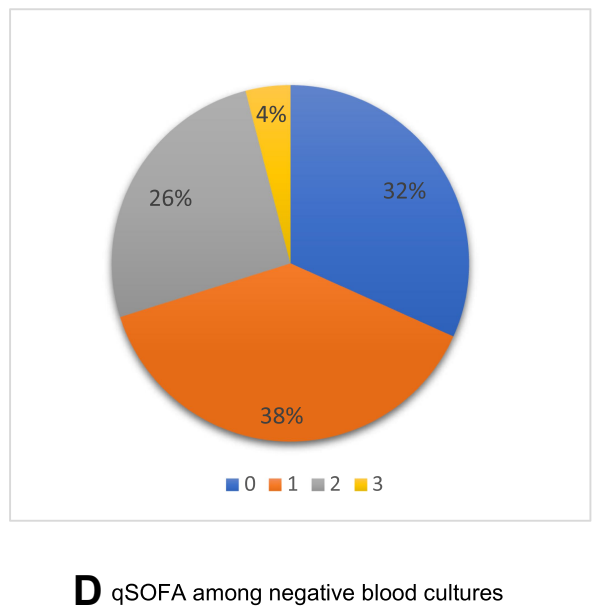

Figure I SIRS and qSOFA scores distribution among positive and negative blood culture.

3. Important statistical factors, like a large number of missing values of some of the lab work that physicians usually do not order when suspecting bacteremia such as (Procalcitonin level - lactic acid and other inflammatory markers), which may have a role in prediction. ${ }^{30,31}$

\section{So, What are the Best Predictors for Blood Culture and When Should We Request Blood Culture?}

While fever or leukocytosis is the major clinical driver for the physician to request for blood culture, several previous studies have shown a lack of correlation between these clinical parameters and bacteremia, ${ }^{19,32,33}$ which this study confirms.

It is not surprising that the presence of central-line, which is a known risk for CLABSI, as a risk factor for bacteremia, a cumulative risk pattern (between1.1-4.8 per 1000 catheterdays). ${ }^{34}$ However, hospitalization exceeding 16 days has been shown as a strong predictor of bacteremia by various models. Therefore, though this finding is not novel, ${ }^{20}$ the majority of the previous studies, including Nielsen et $\mathrm{al}^{24}$ did not show such association as this study has shown in and reflected a community-acquired rather than nosocomial bacteremia.

\section{What is Next?}

While our study used a large sample size and an extensive analysis of the variables by different methods of machine learning, it validated the previous scores, including the majority of the variables/scores which were thought to be related based on the previous work. Not including some clinical assessment, which is compatible with the machine-learning idea and eliminating the variability and the bias in the assessment of some findings (such as suspected endocarditis, nausea or vomiting, and chills) may be a strength and a limitation of this study at the same time. 
Our study has some potential limitations including missing some variables, which may lead to underestimation of some possible associations; inability to assess the risk from the blood culture and which could help to stratify the risk; and, lastly, the large percentage of the population being on antibiotics may change the hosts' response to bacteremia.

\section{Conclusion}

Although Bacteremia is extremely complex and poorly understood, our study provides valuable insights into the predictors of bacteremia such as the duration of hospitalization as meriting attention. The machine-learning performance showed excellent specificity but still needs to improve its sensitivity.

\section{Ethics And Consent}

The study was approved by the Institutional Review Board (IRB) in King Abdullah International Medical Research Center (KAIMRC) (Protocol number RC19/325/R). Written informed consent was waived by the IRB, as the study was a retrospective chart review where research involves no more than minimal risk to the subjects. The study complied with the Declaration of Helsinki concerning maintaining the confidentiality of the patient's data as the data were anonymized.

\section{Disclosure}

The authors report no conflicts of interest in this work.

The authors received no financial support for the research, authorship, and/or publication of this article.

\section{References}

1. Goto M, Al-Hasan MN. Overall burden of bloodstream infection and nosocomial bloodstream infection in North America and Europe. Clin Microbiol Infect. 2013;19(6):501-509. doi:10.1111/1469-0691.12195

2. Kuderer NM, Dale DC, Crawford J, Cosler LE, Lyman GH. Mortality, morbidity, and cost associated with febrile neutropenia in adult cancer patients. Cancer. 2006;106(10):2258-2266. doi:10.1002/cncr.21847

3. Micek ST, Lloyd AE, Ritchie DJ, Reichley RM, Fraser VJ, Kollef MH. Pseudomonas aeruginosa bloodstream infection: importance of appropriate initial antimicrobial treatment. Antimicrob Agents Chemother. 2005;49(4):1306-1311. doi:10.1128/AAC.49.4.1306-1311.2005

4. Paesmans M, Klastersky J, Maertens J, et al. Predicting febrile neutropenic patients at low risk using the MASCC score: does bacteremia matter? Support Care Cancer. 2011;19(7):1001-1008. doi:10.1007/ s00520-010-0925-7

5. Troyanskaya $\mathrm{O}$, Cantor M, Sherlock G, et al. Missing value estimation methods for DNA microarrays. Bioinformatics. 2001;17(6):520-525. doi:10.1093/bioinformatics/17.6.520

6. Analytical Methods C. Robust statistics - how not to reject outliers. Part 1. Basic concepts. Analyst. 1989;114(12):1693-1697.
7. Chawla NV, Bowyer KW, Hall LO, Kegelmeyer WP. SMOTE: synthetic minority over-sampling technique. J Artif Int Res. 2002;16 (1):321-357.

8. Lemaitre G, Nogueira F, Aridas CK. Imbalanced-learn: a python toolbox to tackle the curse of imbalanced datasets in machine learning. J Mach Learn Res. 2017;18(1):559-563.

9. Breiman L. Random forests. Mach Learn. 2001;45(1):5-32. doi:10.1023/A:1010933404324

10. Fan R-E, Chang K-W, Hsieh C-J, Wang X-R, Lin C-J. LIBLINEAR: a library for large linear classification. $J$ Mach Learn Res. 2008;9:1871-1874.

11. Quinlan JR. Induction of decision trees. Mach Learn. 1986;1 (1):81-106. doi:10.1007/BF00116251

12. McCallum A. A comparison of event models for naive Bayes text classification. AAAI Workshop. 1998;1998.

13. Abadi M, Barham P, Chen J, et al. TensorFlow: a system for large-scale machine learning. Proceedings of the 12th USENIX conference on Operating Systems Design and Implementation; 2016; Savannah, GA, USA.

14. Wu T-F, Lin C-J, Weng RC. Probability estimates for multi-class classification by pairwise coupling. $J$ Mach Learn Res. 2004;5:975-1005.

15. Pedregosa F, Varoquaux G, Gramfort A, et al. Scikit-learn: machine learning in python. J Mach Learn Res. 2011;12(null):2825-2830.

16. Bates DW, Cook EF, Goldman L, Lee TH. Predicting bacteremia in hospitalized patients. A prospectively validated model. Ann Intern Med. 1990;113(7):495-500. doi:10.7326/0003-4819-113-7-495

17. Laukemann S, Kasper N, Kulkarni P, et al. Can we reduce negative blood cultures with clinical scores and blood markers? Results from an observational cohort study. Medicine (Baltimore). 2015;94(49): e2264-e2264. doi:10.1097/MD.0000000000002264

18. Shapiro NI, Wolfe RE, Wright SB, Moore R, Bates DW. Who needs a blood culture? A prospectively derived and validated prediction rule. $J$ Emerg Med. 2008;35(3):255-264. doi:10.1016/j. jemermed.2008.04.001

19. Coburn B, Morris AM, Tomlinson G, Detsky AS. Does this adult patient with suspected bacteremia require blood cultures? JAMA. 2012;308(5):502-511. doi:10.1001/jama.2012.8262

20. Jaimes F, Arango C, Ruiz G, et al. Predicting bacteremia at the bedside. Clin Infect Dis. 2004;38(3):357-362. doi:10.1086/380967

21. Paul M, Andreassen S, Nielsen AD, et al. Prediction of bacteremia using TREAT, a computerized decision-support system. Clin Infect Dis. 2006;42(9):1274-1282. doi:10.1086/503034

22. Bouza E, Sousa D, Rodríguez-Créixems M, Lechuz JG, Muñoz P. Is the volume of blood cultured still a significant factor in the diagnosis of bloodstream infections? J Clin Microbiol. 2007;45(9):2765-2769. doi:10.1128/JCM.00140-07

23. Fabre V, Sharara SL, Salinas AB, Carroll KC, Desai S, Cosgrove SE. Does this patient need blood cultures? A scoping review of indications for blood cultures in adult non-neutropenic inpatients. Clin Infect Dis. 2020;71(5):1339-1347. doi:10.1093/cid/ciaa039

24. Nielsen SL, Lassen AT, Kolmos HJ, Jensen TG, Gradel KO, Pedersen $\mathrm{C}$. The daily risk of bacteremia during hospitalization and associated 30-day mortality evaluated in relation to the traditional classification of bacteremia. Am J Infect Control. 2016;44 (2):167-172. doi:10.1016/j.ajic.2015.09.011

25. Wildi K, Tschudin-Sutter S, Dell-Kuster S, Frei R, Bucher HC, Nüesch R. Factors associated with positive blood cultures in outpatients with suspected bacteremia. Eur J Clin Microbiol Infect Dis. 2011;30(12):1615-1619. doi:10.1007/s10096-011$1268-0$

26. Bone RCBR, Cerra FB, Dellinger RP, Fein AM, Knaus WA. American College of Chest Physicians/Society of Critical Care Medicine Consensus Conference: definitions for sepsis and organ failure and guidelines for the use of innovative therapies in sepsis. Crit Care Med. 1992;20(6):864-874. 
27. Kaukonen K-M, Bailey M, Suzuki S, Pilcher D, Bellomo R. Mortality related to severe sepsis and septic shock among critically ill patients in Australia and New Zealand, 2000-2012. JAMA 2014;311(13):1308-1316. doi:10.1001/jama.2014.2637

28. Rodríguez A, Martín-Loeches I, Yébenes JC. New definition of sepsis and septic shock: what does it give us? Med Intensiva. 2017;41(1):38-40. doi:10.1016/j.medin.2016.03.008

29. Giannini HM, Ginestra JC, Chivers C, et al. A machine learning algorithm to predict severe sepsis and septic shock: development, implementation, and impact on clinical practice. Crit Care Med. 2019;47(11):1485-1492. doi:10.1097/CCM.0000000000003891

30. Arai T, Kumasaka K, Nagata K, et al. Prediction of blood culture results by measuring procalcitonin levels and other inflammatory biomarkers. Am J Emerg Med. 2014;32(4):330-333. doi:10.1016/j. ajem.2013.12.035

31. Ratzinger F, Haslacher H, Perkmann T, et al. Machine learning for fast identification of bacteraemia in SIRS patients treated on standard care wards: a cohort study. Sci Rep. 2018;8(1):12233. doi:10.1038/ s41598-018-30236-9
32. Linsenmeyer K, Gupta K, Strymish JM, Dhanani M, Brecher SM, Breu AC. Culture if spikes? Indications and yield of blood cultures in hospitalized medical patients. J Hosp Med. 2016;11(5):336-340. doi:10.1002/jhm.2541

33. Seigel TA, Cocchi MN, Salciccioli J, et al. Inadequacy of temperature and white blood cell count in predicting bacteremia in patients with suspected infection. J Emerg Med. 2012;42(3):254-259. doi:10.1016/ j.jemermed.2010.05.038

34. Maki DG, Kluger DM, Crnich CJ. The risk of bloodstream infection in adults with different intravascular devices: a systematic review of 200 published prospective studies. Mayo Clin Proc. 2006;81 (9):1159-1171.

35. Singer M, Deutschman CS, Seymour CW, et al. The third international consensus definitions for sepsis and septic shock (Sepsis-3). JAMA. 2016;315(8):801-810. doi:10.1001/jama.2016.0287
Infection and Drug Resistance

\section{Publish your work in this journal}

Infection and Drug Resistance is an international, peer-reviewed openaccess journal that focuses on the optimal treatment of infection (bacterial, fungal and viral) and the development and institution of preventive strategies to minimize the development and spread of resistance. The journal is specifically concerned with the epidemiology of
Dovepress

antibiotic resistance and the mechanisms of resistance development and diffusion in both hospitals and the community. The manuscript management system is completely online and includes a very quick and fair peerreview system, which is all easy to use. Visit http://www.dovepress.com/ testimonials.php to read real quotes from published authors. 